Participant flow

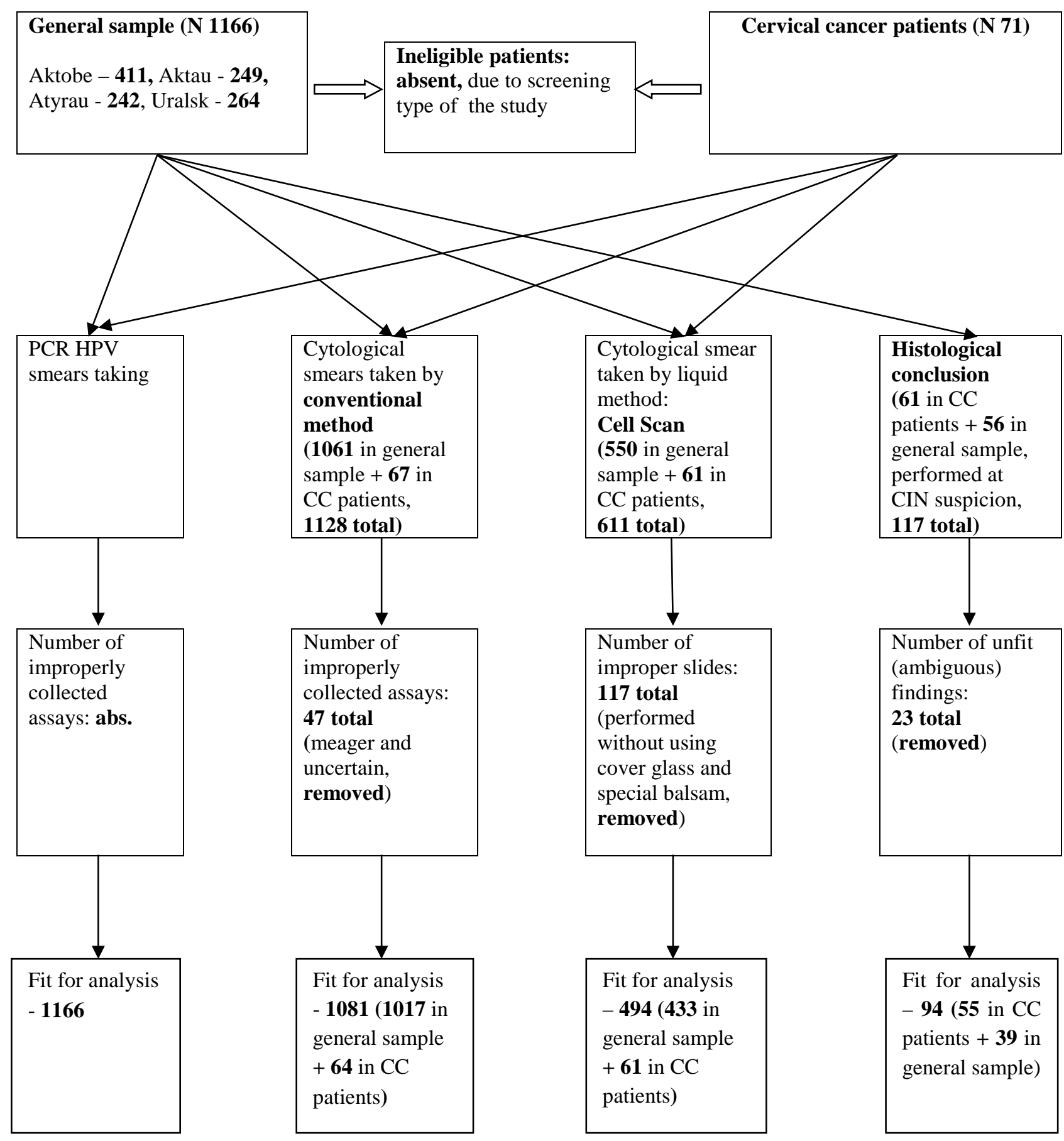


Baseline characteristics

\begin{tabular}{|c|c|c|c|}
\hline \multicolumn{2}{|l|}{ Characteristics } & $\begin{array}{l}\text { Women from general } \\
\text { population } \\
\text { (total } 1166 \text { ) }\end{array}$ & $\begin{array}{l}\text { Women from the group } \\
\text { of Cervical cancer } \\
\text { (total 71) }\end{array}$ \\
\hline \multirow[t]{4}{*}{ Age categories } & $18-29$ & $37.7 \%$ & $1.5 \%$ \\
\hline & $30-39$ & $34.0 \%$ & $21.9 \%$ \\
\hline & $40-49$ & $17.8 \%$ & $34.4 \%$ \\
\hline & $50-60+$ & $10.5 \%$ & $42.2 \%$ \\
\hline \multicolumn{2}{|c|}{ Average age of surveyed } & $\begin{array}{l}34.5 \pm 9.9 \text { (range } 16-63) \\
\text { M } 33.0 \text { (27.0-41.0 } \\
\text { by 25/75 quartile) }\end{array}$ & 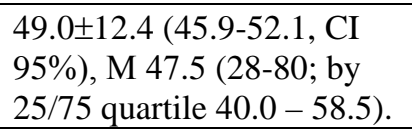 \\
\hline \multirow[t]{3}{*}{ Ethnicity } & Asian & $85.3 \%$ & $79.7 \%$ \\
\hline & European & $13.6 \%$ & $20.3 \%$ \\
\hline & Other (Caucasian) & $1.1 \%$ & - \\
\hline \multirow[t]{3}{*}{ Education level } & School & $31.4 \%$ & $65.6 \%$ \\
\hline & College & $22.9 \%$ & $17.2 \%$ \\
\hline & University & $45.7 \%$ & $17.2 \%$ \\
\hline \multirow[t]{4}{*}{ Employment } & Not occupied & $33.6 \%$ & $48.4 \%$ \\
\hline & Low-skilled labor & $13.7 \%$ & $26.5 \%$ \\
\hline & $\begin{array}{l}\text { Representatives of the } \\
\text { common sector }\end{array}$ & $20.7 \%$ & $9.4 \%$ \\
\hline & Highly qualified work & $32.0 \%$ & $15.6 \%$ \\
\hline \multirow{3}{*}{$\begin{array}{l}\text { (Income per } \\
\text { capita) }\end{array}$} & $(\leq 100 \$$ up to $200 \$$ & $40.1 \%$ & $17.2 \%$ \\
\hline & $200-500 \$$ & $39.4 \%$ & $32.8 \%$ \\
\hline & $500-1000 \$$ and $>$ & $20.5 \%$ & $3.1 \%$ \\
\hline \multirow{3}{*}{$\begin{array}{l}\text { Total number of } \\
\text { pregnancies }\end{array}$} & none & $10.7 \%$ & $3.1 \%$ \\
\hline & $1-2$ & $36.1 \%$ & $25.0 \%$ \\
\hline & 3 and more & $53.2 \%$ & $71.9 \%$ \\
\hline \multicolumn{2}{|c|}{$\begin{array}{l}\text { Average number of pregnancies } \\
\text { in the history }\end{array}$} & $\begin{array}{l}3.0 \pm 2.2 \text { (range } 0-16 \text { ) } \\
\text { M } 3.0 \text { (2.8-4.4 by } 25 / 75 \\
\text { quartile) }\end{array}$ & \\
\hline \multirow{2}{*}{$\begin{array}{l}\text { Hereditary factor: } \\
\text { presence of close } \\
\text { relatives with } \\
\text { cervical cancer }\end{array}$} & Yes & $5.1 \%$ & $9.4 \%$ \\
\hline & No & $94.9 \%$ & $90.6 \%$ \\
\hline \multicolumn{2}{|c|}{ Age of onset of sexual activity* } & $\begin{array}{l}20.8 \pm 3.4 \text { (range } 13-45 \text { ) } \\
\text { M } 20.0 \text { (18.0-22.0 by } \\
25 / 75 \text { quartile) }\end{array}$ & $\begin{array}{l}\text { 20.3 } 2.3 \text { (19.4 - 20.8;CI } \\
\text { 95\%), M } 20.0 \text { (range 15-27; } \\
\text { 25/75 quartile 19.0 - 21.0). }\end{array}$ \\
\hline \multirow{3}{*}{$\begin{array}{l}\text { Experience of } \\
\text { sexual life }\end{array}$} & $0-10$ years & $47.2 \%$ & $3.1 \%$ \\
\hline & $11-20$ years & $29.9 \%$ & $31.3 \%$ \\
\hline & More than 20 years & $22.9 \%$ & $65.6 \%$ \\
\hline \multicolumn{2}{|c|}{ The average experience of sexual life } & $\begin{array}{l}13.5 \pm 9.2 \text { (range } 1-45) \\
\text { M } 12.0 \text { (6.0-20.0 by } 25 / 75 \\
\text { quartile) }\end{array}$ & $\begin{array}{l}\text { 26.5 } \pm 10.8 \text { (23.3 - 29.7; CI } \\
95 \%), \text { M } 22.0 \text { (range 7-59). }\end{array}$ \\
\hline \multirow{3}{*}{$\begin{array}{l}\text { Number of } \\
\text { sexual partners } \\
\text { during life } \\
\end{array}$} & 1 partner & $64.7 \%$ & $60.9 \%$ \\
\hline & $2-5$ partners & $28.2 \%$ & $28.1 \%$ \\
\hline & 6 and more & $7.1 \%$ & $10.8 \%$ \\
\hline \multicolumn{2}{|c|}{ Average number of sexual partners during life } & $\begin{array}{l}2.2 \pm 2.9 \text { (range } 1-30) \\
(1.9 ; 2.7 \text { CI 95\%) }\end{array}$ & $\begin{array}{l}3.0 \pm 3.4 \text { (2.1 - 3.9; CI 95\%), } \\
\text { M } 1.0 \text { (range 1-15). }\end{array}$ \\
\hline $\begin{array}{l}\text { Application of } \\
\text { BCP }\end{array}$ & Birth control pills & $4.8 \%$ & - \\
\hline \multirow{2}{*}{$\begin{array}{l}\text { Attitude towards } \\
\text { smoking* }\end{array}$} & Smoking * & $10.8 \%$ & $9.4 \%$ \\
\hline & Do not smoke & $89.2 \%$ & $90.6 \%$ \\
\hline \multirow{3}{*}{$\begin{array}{l}\text { Attendance of the } \\
\text { state-sponsored } \\
\text { PHC at the place } \\
\text { of residence }\end{array}$} & Visits constantly & $40.7 \%$ & $31.3 \%$ \\
\hline & $\begin{array}{l}\text { Visits sometimes, } \\
\text { irregularly }\end{array}$ & $46.3 \%$ & $39.0 \%$ \\
\hline & $\begin{array}{l}\text { Does not visit, only private } \\
\text { medical facilities }\end{array}$ & $13.0 \%$ & $29.7 \%$ \\
\hline
\end{tabular}




\begin{tabular}{|c|c|c|c|}
\hline \multirow{3}{*}{$\begin{array}{l}\text { Participation in } \\
\text { state screening } \\
\text { program for } \\
\text { cervical cancer }\end{array}$} & Constantly participates & $34.7 \%$ & $39.0 \%$ \\
\hline & $\begin{array}{l}\text { Participates } \\
\text { irregularly }\end{array}$ & $28.0 \%$ & $15.6 \%$ \\
\hline & $\begin{array}{l}\text { Does not participate - } \\
\text { ignores in favor of private } \\
\text { clinics }\end{array}$ & $37.3 \%$ & $45.3 \%$ \\
\hline \multirow{4}{*}{$\begin{array}{l}\text { Awareness of } \\
\text { vaccination } \\
\text { against cervical } \\
\text { cancer }\end{array}$} & $\begin{array}{l}\text { Knows nothing about } \\
\text { vaccination }\end{array}$ & $38.8 \%$ & $60.9 \%$ \\
\hline & $\begin{array}{l}\text { Heard about vaccination, } \\
\text { but does not know how to } \\
\text { treat }\end{array}$ & $33.6 \%$ & $25.0 \%$ \\
\hline & $\begin{array}{l}\text { Welcomes vaccination } \\
\text { against cervical cancer }\end{array}$ & $22.9 \%$ & $10.9 \%$ \\
\hline & $\begin{array}{l}\text { Set against vaccination } \\
\text { (consider unnecessary and } \\
\text { dangerous) }\end{array}$ & $4.7 \%$ & $3.1 \%$ \\
\hline
\end{tabular}




\section{Outcome measures}

\begin{tabular}{|c|c|c|c|}
\hline \multicolumn{2}{|l|}{ Primary outcomes } & $\begin{array}{l}\text { Women from general } \\
\text { population } \\
\text { (total 1166) }\end{array}$ & $\begin{array}{l}\text { Women from the } \\
\text { group of Cervical } \\
\text { cancer (total 71) }\end{array}$ \\
\hline \multicolumn{2}{|c|}{$\begin{array}{l}\text { 1. Total HPV prevalence and in the each province of the } \\
\text { region: }\end{array}$} & \multicolumn{2}{|c|}{$\begin{array}{l}\text { Total \% across the region }-24.9 \%(22.3 ; 27.7 \text { CI } 95 \% \text {, } \\
\mathrm{p}=0.05) ; \\
\text { Mangystau - } 19.3 \text { (CI 95\% 14.5;24.2) } \mathrm{p}=0.049 ; \\
\text { Aktobe - } 23.9(19.7 ; 28.6) \mathrm{p}=0.044 ; \\
\text { Atyrau - } 28.0(22.8 ; 34.1) \mathrm{p}=0.056 ; \\
\text { Uralsk - } 29.5(24.0 ; 34.9) \mathrm{p}=0.054\end{array}$} \\
\hline \multicolumn{2}{|c|}{$\begin{array}{l}\text { 2. Average viral load in HPV-infected, both in general } \\
\text { population and in CC diseased: }\end{array}$} & $\begin{array}{l}5.5 \pm 3.8(0.9-22.3 ; \text { CI } 95 \% \\
5.1 ; 5.9)\end{array}$ & $\begin{array}{l}6.9 \pm 4.0(3.1-19.5 ; \text { CI } 95 \% \\
6.1 ; 7.7)\end{array}$ \\
\hline \multicolumn{2}{|c|}{$\begin{array}{l}\text { 2. HPV leading types in general population and in CC } \\
\text { diseased: }\end{array}$} & $\begin{array}{l}\text { type } 16-26.4 \% \\
\text { type } 31-10.1 \% \\
\text { type } 51-9.4 \% \\
\text { type } 52-9.0 \% \\
\text { type } 6-7.9 \%\end{array}$ & $\begin{array}{l}\text { type } 16-71.9 \% \\
\text { type } 31-14.1 \% \\
\text { type } 58-7.8 \% \\
\text { type } 18-7.8 \% \\
\text { type } 33-6.2 \%\end{array}$ \\
\hline \multirow{4}{*}{$\begin{array}{l}\text { 4. \% of women infected } \\
\text { HR-HPV genotypes in } \\
\text { each age categories }\end{array}$} & $18-29$ & 26.7 (p 0.003) & \\
\hline & $30-39$ & $21.1(\mathrm{p} 0.013)$ & \\
\hline & $40-49$ & $9.8(\mathrm{p} 0.004)$ & \\
\hline & $50-60+$ & $8.2(\mathrm{p} 0.019)$ & \\
\hline \multicolumn{2}{|c|}{$\begin{array}{l}\text { 5. \% and distribution of HPV different types in normal } \\
\text { cytology; }\end{array}$} & \multicolumn{2}{|c|}{$\begin{array}{l}\text { Total \% - 44.8; } \\
\text { leading types - } 16 \text { (30.8\%); } 31-13.5 \% \text {; } 6 \text { - 11.5\%; } 66 \\
-9.6 \% \text {; } 51-7.7 \% \text {. }\end{array}$} \\
\hline \multirow{8}{*}{\multicolumn{2}{|c|}{$\begin{array}{l}\text { 6. The most significant risk factors for the CC } \\
\text { implementation: }\end{array}$}} & \multirow{2}{*}{\multicolumn{2}{|c|}{$\begin{array}{l}\text { Age 50-60+ (p-value 0.003, Cramer's V 0.29) } \\
\text { Education - school level (p-value 0.00075, Cramer's V } \\
0.33 \text { ) }\end{array}$}} \\
\hline & & & \\
\hline & & \multicolumn{2}{|c|}{$\begin{array}{l}\text { Occupation - not occupied (p-value } 0.00053 \text {, Cramer's } \\
\text { V 0.37) }\end{array}$} \\
\hline & & \multicolumn{2}{|c|}{$\begin{array}{l}\text { Income per capita - below or barely exceeding the } \\
\text { subsistence level (p-value } 0.001 \text {, Cramer's V 0.33) }\end{array}$} \\
\hline & & \multicolumn{2}{|c|}{$\begin{array}{l}\text { Experience of sexual life - more than } 20 \text { years (p-value } \\
0.007 \text {, Cramer's V 0.28) }\end{array}$} \\
\hline & & \multicolumn{2}{|c|}{$\begin{array}{l}\text { Participation in screening programmes - non- } \\
\text { participating (p-value } 0.006 \text {, Cramer’s V } 0.28 \text { ) }\end{array}$} \\
\hline & & \multicolumn{2}{|c|}{$\begin{array}{l}\text { Vaccination awareness - fully not aware (p-value } \\
0.026 \text {, Cramer's V 0.27) }\end{array}$} \\
\hline & & \multicolumn{2}{|c|}{ Presence of HPV type 16 - (p-value 0.00007 , Phi 0.35 ) } \\
\hline \multirow{2}{*}{\multicolumn{2}{|c|}{ 7. Probability of the Cervical cancer decrease (ORs): }} & \multicolumn{2}{|c|}{$\begin{array}{l}\text { Income per capita - off-being category } \\
(0.0713, \text { CI } 95 \%-0.0130-0.3912)\end{array}$} \\
\hline & & \multicolumn{2}{|c|}{$\begin{array}{l}\text { Participation in screening programmes - even though } \\
\text { irregular (sometimes) participating } \\
(0.3384 \text {, CI } 95 \%-0.1269-0.9023)\end{array}$} \\
\hline
\end{tabular}

\section{Secondary outcomes}

1. Results of comparison of two cytological methods operating in the region

(amount of non-informative material by each method; degree of concordance with histological conclusions in each method;

results of ROC analysis;

Kappa statistics)

2. Colposcopic data:

correlation between HPV viral load and points RCI

(Reid colposcopic index)

3. Overall trend of CC morbidity in the region and in
Non-informative material by each method:

CellScan $-5.9 \%$ vs. $0.4 \%$ by Romanovsky-Giemsa

Concordance with histological conclusions:

CellScan $-61.7 \%$ vs. $60.6 \%$ by Romanovsky-Giemsa

Data on ROC-analysis:

CellScan - AUC 0.84 vs. AUC 0.92 by Romanovsky-

Giemsa

Kappa statistics:

CellScan $-\kappa=0.47$ vs. 0.62 by Romanovsky-Giemsa

Spierman's $r=0.316$, p-value -0.0000

Overall trend tends to increase with the rate of growth 


\begin{tabular}{|c|c|c|}
\hline \multicolumn{2}{|l|}{ each age categories } & $\begin{array}{l}\text { Tpr }=3.2 \% \text {. } \\
\text { In } 18-29 \text { years category - } \mathrm{Tpr}=-3.1 \% \text {; } \\
\text { in } 30-39-\mathrm{Tpr}=-6.2 \% \text {; } \\
\text { in } 40-49-\mathrm{Tpr}=5.5 \% \text {; } \\
\text { in } 50-59-\mathrm{Tpr}=0.9 \% \text {; } \\
\text { in } 60-69-\mathrm{Tpr}=3.6 \% \text {. } \\
\text { in } 70+-\mathrm{Tpr}=13.6 \% \text {. }\end{array}$ \\
\hline \multirow[t]{10}{*}{$\begin{array}{l}\text { 4. Detection of various HPV gene } \\
\text { L1 isolates circulating in the region }\end{array}$} & Aktau & $\begin{array}{l}\text { The isolate of genotype 18, similar to KC470221.1 } \\
\text { isolate, country - the USA }\end{array}$ \\
\hline & Aktobe, Alga & $\begin{array}{l}\text { An authentic isolate for the region, not represented in the } \\
\text { world genebank. }\end{array}$ \\
\hline & Aktau & Isolate KC706450.1 from Saudi Arabia. \\
\hline & Aktobe & $\begin{array}{l}\text { A sequence is obtained, similar to EU918764 isolate, the } \\
\text { country is China. }\end{array}$ \\
\hline & Uralsk & $\begin{array}{l}\text { An isolate forming a remote cluster with KF436822 / 1, } \\
\text { KU951264.1 - Southwest China, 97\% identity with } \\
\text { EU056643.1 - Ireland was obtained. }\end{array}$ \\
\hline & $\begin{array}{l}\text { Khromtau, } \\
\text { Aktobe }\end{array}$ & $\begin{array}{l}\text { An isolate similar to KU951264 /1 was obtained, } \\
\text { forming a separate cluster with } \\
\text { AJ617545.1, the country of origin of both isolates is } \\
\text { Cyprus. }\end{array}$ \\
\hline & $\begin{array}{l}\text { Khromtau, } \\
\text { Aktobe }\end{array}$ & $\begin{array}{l}\text { Common cluster with isolates KU707481.1 (country - } \\
\text { Netherlands), } \\
\text { GQ465900 (country - Canada). }\end{array}$ \\
\hline & Aktobe, Shalkar & $\begin{array}{l}\text { An isolate of genotype } 16 \text { similar to isolate AJ617545.1 } \\
\text { was obtained. } \\
\text { Country - Republic of Cyprus }\end{array}$ \\
\hline & Aktobe, Temir & $\begin{array}{l}\text { An isolate of genotype } 16 \text { similar to isolate EF133498.1 } \\
\text { was obtained. } \\
\text { Country - Portugal. }\end{array}$ \\
\hline & Aktobe, Kayndy & $\begin{array}{l}\text { An isolate of genotype } 31 \text {, similar to isolate } \\
\text { KX514424.1, was obtained. } \\
\text { Country - Brazil }\end{array}$ \\
\hline
\end{tabular}


Adverse events

There were no adverse events associated with this study due to the absence of interventions. The study constituted a kind of screening and all enrolled were being interviewed and examined by commonly accepted methods during one-time visit. 